\title{
Cooperative Human Tissue Network
}

National Cancer Institute

\section{Source}

National Cancer Institute. Cooperative Human Tissue Network. NCI Thesaurus. Code C19328.

Specializes in the prospective procurement, preservation and distribution of human tissues for research. Trained personnel coordinate the retrieval, preservation and delivery of specimens obtained from surgical resections and from autopsies. This resource provides normal, benign, pre-cancerous and cancerous human tissue to the scientific community for basic and developmental studies in many areas of cancer research. 